\title{
On the Possibility of Landau-Pekar Triplet Bipolaron Existance
}

\author{
Vladimir Mukhomorov
}

Agrophysical Institute, St-Petersburg, 195220, Russia

\begin{abstract}
A theoretical analysis of a homogeneous magnetic field the action on the electronic terms of the bipolaron in the triplet $2^{3} P_{z, x \pm i y}$ state is made. It is shown that the action of the magnetic field, on the one hand, leads to displacement of the triplet terms to the low-energy region and thus to stabilization of the bipolaron and, on the other hand, appreciable weakens the criteria that limit the conditions of their existence. It is shown that the inclusion of electron-electron correlation leads to the stabilization of the triplet bipolaron, as well as to expand the boundaries of his existence.
\end{abstract}

Keywords Bipolaron, Polaron, Singlet, Triplet, Magnetic Field, Interelectron Correlations

\section{Introduction}

It was postulated previously[1] the possibility of the existence in polar media of a bound two polarons (bipolaron) formations in a trip let $\left(2^{3} P\right)$ state whose structure resembles the helium atom in the excited $1 s 2 p_{z, x \pm i y}$ state. Such two polarons species can be formed in polar media where autolocalization is associated with the formation of a fairly deep and wide polarization potential well. It was shown that polarons can exist in the ground and relaxation-excited states. As is well known[2], optical excitation of a polaron Franck-Condon transition occurs between the electronic $1 \mathrm{~s}$ and $2 p_{z, x \pm i y}$ - states, the oscillator strength is $\sim 0.9$. At the same time the possible existence of singlet axially- symmetrical bipolarons has been widely discussed[3-10]. The most probable dipole-actives the optical transition for ground state of Landau-Pekar bipolaron will be ${ }^{1} \sum\left(1 s^{2}\right) \rightarrow{ }^{1} \sum(1 s 2 p)$. For the special case $\varepsilon^{*} / \varepsilon_{\infty}=1.075$ (a polaron in ammonia), we obtain the transition frequency $\hbar \Omega=0.82 \mathrm{eV}$, which is very close to the experimental value $0.81 \mathrm{eV}[10]$.

The bipolarons in triplet state will have an anomalous chemical activity and, in view of the translational invariance of the system, can transfer the trapped energy quantum in a selected direction. The spin prohibition of the emission of a photon from a metastable state is lifted as a result of relativistic interactions, but this deactivation mechanism is inefficient. A lso possible are $T \rightarrow S$ (triplet $\rightarrow$ singlet) transition due to the interaction of the electron spin with the transverse phonons of the polar media. They lead to a change in the

* Corresponding author:

vmukhomorov@mail.ru (Vladimir Mukhomorov)

Published online at http://journal.sapub.org/ajcmp

Copyright (C) 2012 Scientific \& Academic Publishing. All Rights Reserved spatial symmetry of the bipolaron. From a centrally symetric state it changes to a singlet quasi-molecular bipolaron. However, in view of the small magnitude of the magnetic interactions, the spontaneous $T \rightarrow S$ transitions are of low probability. Triplet bipolarons may be treated as active centers having an inverted population relative to the ground singlet state.

It is known[11] that a magnetic field lowers the energy of a polarons and stabilizes the re laxation-excited states relative to the processes of nonradiative deactivation. The present paper analyzes the conditions of triplet bipolarons existence and the effect of a magnetic field on the energy terms bipolaron in trip let state.

\section{Basic Equations and Mathematical Method}

In the adiabatic approximation the wave function of the system, the electron and the field of longitudinal longwavelength phonons of polar medium can be described as the product

$$
\Psi=\chi\left(\mathbf{r}_{1}, \mathbf{r}_{2}\right) \Phi
$$

of a two-electron wave function $\chi\left(\mathbf{r}_{1}, \mathbf{r}_{2}\right)$ and a wave function $\Phi$ of normal vibrations of a dielectric continuum. To determine the energy of the two polarons system it is necessary to minimize the functional

$$
F=\langle\Psi|\mathrm{H}| \Psi\rangle,
$$

under the additional conditions $\langle\chi \mid \chi\rangle=1$ and $\langle\Phi \mid \Phi\rangle=1$; to describe the behavior of electrons in a polarizing medium we use the generalized Hamiltonian

$$
\mathrm{H}=H-\mathrm{v}_{z}\left(P_{z}-\hbar K_{z}\right),
$$

where $P_{z}$ is the component of the total mo mentu moperator, $K_{z}$ is its eigenvalue, and $\mathrm{v}_{z}$ is the mean translation velocity 
of the polaron moving along the $z$ axis,

$$
\begin{gathered}
H=\frac{1}{2 m^{*}} \sum_{j=1,2}\left(-i \hbar \nabla_{j}-\frac{e}{c} \mathbf{A}_{j}\right)^{2}+ \\
+\sum_{\mathbf{q}, j=1,2}\left[V_{\mathbf{q}} b_{\mathbf{q}} \exp \left(i \mathbf{q} \mathbf{r}_{j}\right)+V_{\mathbf{q}}^{*} b_{\mathbf{q}}^{+} \exp \left(-i \mathbf{q} \mathbf{r}_{j}\right)\right], \\
+\frac{e^{2}}{\varepsilon_{\infty} r_{12}}+\sum_{q} \hbar \omega_{0} b_{\mathbf{q}}^{+} b_{\mathbf{q}},
\end{gathered}
$$

where $\mathbf{r}_{j}$ is the radius vector of the $j$ th electrons; $b_{\mathbf{q}}$ and $b_{\mathbf{q}}{ }^{+}$ are the Bose operators of annihilation and creation of a quantum of longitudinal phonons with quasi-momentum $\hbar \mathbf{q}$ and longitudinal eigenfrequency $\omega_{0}(q=0)$; and the Fourier coefficients $V_{\mathbf{q}}=i\left(\alpha_{c} 4 \pi / V\right)^{1 / 2} \hbar \omega_{0} \quad\left(\hbar / 2 m^{*} \omega_{0}\right)^{1 / 4} \times$ $|\mathbf{q}|^{-1}$ characterize the interaction of the electron with the polarization continuum. The dimensionless coupling constant is $\alpha_{c}=\left(e^{2} / 2 \hbar \omega_{0}\right)\left(2 m^{*} \omega_{0} / \hbar\right)^{1 / 2} \varepsilon^{*-1}, m^{*}$ is the effective electron mass in the approximation of the isotropic and quadratic law of dispersion, and $V$ is the volume of the main region of the continuum. The effective permittivity is $\varepsilon^{*}=\varepsilon_{s} \varepsilon_{\infty}\left(\varepsilon_{s}-\varepsilon_{\infty}\right)^{-1}, \quad \varepsilon_{\infty}$ and $\varepsilon_{s}$ are the high-frequency and static permittivities of the polar medium. The vector potential of a constant homogeneous magnetic field directed along the $z$ axis will be chosen in the symmetric form $\mathbf{A}=\left(-\mathrm{H}_{z} y / 2, \mathrm{H}_{z} x / 2,0\right)$.

Varying Eq. (2) with respect to $\Phi$ and allowing for Eqs. (1) and (3), we obtain the following equation for the eigenvalues for the polarization continuum:

$$
\left\langle\chi^{*}\left(\mathbf{r}_{1}, \mathbf{r}_{2}\right)\left|\left[H-v_{z}\left(P_{z}-\hbar K_{z}\right)\right]\right| \chi\left(\mathbf{r}_{1}, \mathbf{r}_{2}\right)\right\rangle \Phi=\varepsilon[\chi] \Phi(5)
$$

The left-hand side of Eq. (5) can be rewritten as follows:

$$
\begin{aligned}
& H=\left\langle\chi^{*}\left(\mathbf{r}_{1}, \mathbf{r}_{2}\right)\left|\frac{1}{2 m^{*}} \sum_{j=1,2}\left(-i \hbar \nabla_{j}-\frac{e}{c} \boldsymbol{A}_{j}\right)^{2}\right| \chi\left(\mathbf{r}_{1}, \mathbf{r}_{2}\right)\right\rangle+ \\
&+\sum_{\mathbf{q}} \hbar\left(\omega_{0}-\mathrm{v}_{z} q_{z}\right) b_{\mathbf{q}}^{+} b_{\mathbf{q}}- \\
&-\mathrm{v}_{z}\left\langle\chi^{*}\left(\mathbf{r}_{1}, \mathbf{r}_{2}\right)\left|\sum_{j=1,2}-i \hbar \frac{\partial}{\partial z_{j}}\right| \chi\left(\mathbf{r}_{1}, \mathbf{r}_{2}\right)\right\rangle+ \\
&+\left\langle\chi^{*}\left(\mathbf{r}_{1}, \mathbf{r}_{2}\right)\left|\frac{e^{2}}{\varepsilon_{\infty} r_{12} \mid}\right| \chi\left(\mathbf{r}_{1}, \mathbf{r}_{2}\right)\right\rangle+\hbar \mathrm{v}_{z} K_{z}+ \\
&+\left\langle\chi^{*}\left(\mathbf{r}_{1}, \mathbf{r}_{2}\right)\right| \sum_{\mathbf{q}, j=1,2}\left[V_{\mathbf{q}} \exp \left(i \mathbf{q} \mathbf{r}_{j}\right) b_{\mathbf{q}}+\right. \\
&\left.+V_{\mathbf{q}}^{*} \exp \left(-i \mathbf{q} \mathbf{r}_{j}\right) b_{\mathbf{q}}^{+}\right]\left|\chi\left(\mathbf{r}_{1}, \mathbf{r}_{2}\right)\right\rangle .
\end{aligned}
$$

We introduce the unitary transformation

$$
S=\exp \left[\sum_{\mathbf{q}}\left(f_{\mathbf{q}} b_{\mathbf{q}}^{+}-f_{\mathbf{q}}^{+} b_{\mathbf{q}}\right)\right],
$$

which converts Eq. (6) to a diagonal form.

The unknown functions $f_{\mathbf{q}}$ and $f_{\mathbf{q}}{ }^{*}$ are determined fro $m$ the condition of minimum of the functional $S^{+} H S$. Considering the operator identities

$$
S^{+} b_{\mathbf{q}} S=b_{\mathbf{q}}-f_{\mathbf{q}}, \quad S^{+} b_{\mathbf{q}}^{+} S=b_{\mathbf{q}}^{+}-f_{\mathbf{q}}
$$

we obtain

$$
\begin{aligned}
& =\left\langle\chi^{*}\left(\mathbf{r}_{1}, \mathbf{r}_{2}\right)\left|\frac{1}{2 m^{*}} \sum_{j=1,2}\left(-i \hbar \nabla_{j}-\frac{e}{c} \boldsymbol{A}_{j}\right)^{2}\right| \chi\left(\mathbf{r}_{1}, \mathbf{r}_{2}\right)\right\rangle+ \\
& +\left\langle\chi^{*}\left(\mathbf{r}_{1}, \mathbf{r}_{2}\right)\right| \sum_{\mathbf{q}, j=1,2}\left[V_{\mathbf{q}} \exp \left(i \mathbf{q} \mathbf{r}_{j}\right)\left(b_{\mathbf{q}}-f_{\mathbf{q}}^{*}\right)+\right. \\
& \left.+V_{\mathbf{q}}^{*} \exp \left(-i \mathbf{q} \mathbf{r}_{j}\right)\left(b_{\mathbf{q}}^{+}-f_{\mathbf{q}}\right)\right]\left|\chi\left(\mathbf{r}_{1}, \mathbf{r}_{2}\right)\right\rangle+ \\
& +\sum_{q} \hbar \varpi\left(b_{\mathbf{q}}^{+} b_{\mathbf{q}}-f_{\mathbf{q}} b_{\mathbf{q}}-b_{\mathbf{q}}^{+} f_{\mathbf{q}}+f_{\mathbf{q}} f_{\mathbf{q}}^{*}\right)+\hbar \mathrm{v}_{z} K_{z}+ \\
& +\left\langle\chi^{*}\left(\mathbf{r}_{1}, \mathbf{r}_{2}\right)\left|\frac{e^{2}}{\varepsilon_{\infty} r_{12}}\right| \chi\left(\mathbf{r}_{1}, \mathbf{r}_{2}\right)\right\rangle- \\
& -\mathrm{v}_{z}\left\langle\chi^{*}\left(\mathbf{r}_{1}, \mathbf{r}_{2}\right)\left|\sum_{j=1,2}-i \hbar \frac{\partial}{\partial z_{j}}\right| \chi\left(\mathbf{r}_{1}, \mathbf{r}_{2}\right)\right\rangle .
\end{aligned}
$$

Varying Eq. (8) with respect to $f_{\mathbf{q}}$ and $f_{\mathbf{q}}{ }^{*}$, we find the unknown functions

$$
f_{\mathbf{q}}=b_{\mathbf{q}}^{+}+\frac{V_{\mathbf{q}} \rho_{\mathbf{q}}}{\hbar \varpi}, f_{\mathbf{q}}=b_{\mathbf{q}}+\frac{V_{\mathbf{q}}^{*} \rho_{\mathbf{q}}^{*}}{\hbar \varpi},
$$

where

$$
\begin{gathered}
\rho_{\mathbf{q}}=<\chi\left(\mathbf{r}_{1}, \mathbf{r}_{2}\right)\left|\sum_{j=1,2} \exp \left(i \mathbf{q} \mathbf{r}_{j}\right)\right| \chi\left(\mathbf{r}_{1}, \mathbf{r}_{2}\right)>, \\
\varpi=\omega_{0}-\mathrm{v}_{z} q_{z} .
\end{gathered}
$$

Substituting Eq.(9) into Eq.(8) we obtain the total energy functional, which takes into account the translational displacement of the bipolaron as a whole

$$
\begin{gathered}
\varepsilon[\chi]=<\chi^{*}\left(\mathbf{r}_{1}, \mathbf{r}_{2}\right)\left|\frac{1}{2 m^{*}} \sum_{j=1,2}\left(-i \hbar \nabla_{j}-\frac{e}{c} \mathbf{A}_{j}\right)^{2}\right| \chi\left(\mathbf{r}_{1}, \mathbf{r}_{2}\right)>- \\
-\sum_{\mathbf{q}} \frac{\left|V_{\mathbf{q}} \rho_{\mathbf{q}}\right|^{2}}{\hbar \varpi}+\hbar \mathrm{v}_{z} K_{z}+ \\
-\mathbf{v}_{z}<\chi^{*}\left(\mathbf{r}_{1}, \mathbf{r}_{2}\right)\left|-i \hbar \sum_{j=1,2} \frac{\partial}{\partial z_{j}}\right| \chi\left(\mathbf{r}_{1}, \mathbf{r}_{2}\right)>+ \\
+<\chi\left(\mathbf{r}_{1}, \mathbf{r}_{2}\right)\left|\frac{e^{2}}{\varepsilon_{\infty} r_{12}}\right| \chi\left(\mathbf{r}_{1}, \mathbf{r}_{2}\right)>.
\end{gathered}
$$

Considering the cylindrical symmetry of the problem, for slow velocity $\mathrm{v}_{z}$ the electronic part of the wave function will be written in the form

$$
\chi\left(\mathbf{r}_{1}, \mathbf{r}_{2}\right)=\chi^{0}\left(\mathbf{r}_{1}, \mathbf{r}_{2}\right) \exp \left[i m^{*} \mathrm{v}_{z}\left(z_{1}+z_{2}\right) / \hbar\right] .
$$

Substituting Eq. (11) into Eq.(10), we obtain the following equation for total energy of two-polaron system

$$
\begin{aligned}
\varepsilon[\chi]=- & \frac{\hbar^{2}}{2 m^{*}}<\chi^{0}\left(\mathbf{r}_{1}, \mathbf{r}_{2}\right)\left|\sum_{j=1,2} \nabla_{j}^{2}\right| \chi^{0}\left(\mathbf{r}_{1}, \mathbf{r}_{2}\right)>- \\
& -m^{*} \mathbf{v}_{z}^{2}+\hbar \mathrm{v}_{z} K_{z}-\sum_{\mathbf{q}} \frac{\left|V_{\mathbf{q}} \rho_{\mathbf{q}}\right|^{2}}{\hbar \varpi}+
\end{aligned}
$$




$$
\begin{gathered}
+\frac{e^{2} H_{z}^{2}}{8 m^{*} c^{2}}<\chi^{0}\left(\mathbf{r}_{1}, \mathbf{r}_{2}\right)\left|\sum_{j=1,2}\left(x_{j}^{2}+y_{j}^{2}\right)\right| \chi^{0}\left(\mathbf{r}_{1}, \mathbf{r}_{2}\right)>- \\
-\frac{i e \hbar H_{z}}{2 m^{*} c}<\chi^{0}\left(\mathbf{r}_{1}, \mathbf{r}_{2}\right)\left|\sum_{j=1,2}\left(x_{j} \frac{\partial}{\partial y_{j}}-y_{j} \frac{\partial}{\partial x_{j}}\right)\right| \chi^{0}\left(\mathbf{r}_{1}, \mathbf{r}_{2}\right)>+ \\
+<\chi^{0}\left(\mathbf{r}_{1}, \mathbf{r}_{2}\right)\left|\frac{e^{2}}{\varepsilon_{\infty} r_{12}}\right| \chi^{0}\left(\mathbf{r}_{1}, \mathbf{r}_{2}\right)>.
\end{gathered}
$$

Assuming that $\hbar K_{z}=\left\langle\Psi\left|P_{z}\right| \Psi\right\rangle$, as well as the definitions Eqs.(1) and (11), for the eigenvalue of the momentum operator, we find

$$
\begin{gathered}
\hbar K_{z}=<\chi^{0}\left(\mathbf{r}_{1}, \mathbf{r}_{2}\right)\left|-i \hbar \sum_{j=1,2} \frac{\partial}{\partial z_{j}}\right| \chi^{0}\left(\mathbf{r}_{1}, \mathbf{r}_{2}\right)>+ \\
+2 m^{*} \mathrm{v}_{z}+\sum_{\mathbf{q}} \frac{q_{z}\left|V_{\mathbf{q}} \rho_{\mathbf{q}}\right|^{2}}{\hbar \varpi^{2}} .
\end{gathered}
$$

Substituting Eq. (13) into Eq. (12), we can readily obtain a general expression for the total energy of a bipolaron in a magnetic field allowing for its translational displacement:

$$
\begin{gathered}
\varepsilon[\chi]=-\frac{\hbar^{2}}{2 m^{*}}<\chi^{0}\left(\mathbf{r}_{1}, \mathbf{r}_{2}\right)\left|\sum_{j=1,2} \nabla_{j}^{2}\right| \chi^{0}\left(\mathbf{r}_{1}, \mathbf{r}_{2}\right)>+m^{*} \mathbf{v}_{z}^{2}+ \\
+\frac{e^{2} H_{z}^{2}}{8 m^{*} c^{2}}<\chi^{0}\left(\mathbf{r}_{1}, \mathbf{r}_{2}\right)\left|\sum_{j=1,2}\left(x_{j}^{2}+y_{j}^{2}\right)\right| \chi^{0}\left(\mathbf{r}_{1}, \mathbf{r}_{2}\right)>+ \\
+\sum_{\mathbf{q}} \frac{\left(2 \mathbf{v}_{z} q_{z}-\omega_{0}\right)\left|V_{\mathbf{q}} \rho_{\mathbf{q}}\right|^{2}}{\hbar \varpi^{2}}- \\
-i \hbar \mathbf{v}_{z}<\chi^{0}\left(\mathbf{r}_{1}, \mathbf{r}_{2}\right)\left|\sum_{j=1,2} \frac{\partial}{\partial z_{j}}\right| \chi^{0}\left(\mathbf{r}_{1}, \mathbf{r}_{2}\right)>+ \\
+<\chi^{0}\left(\mathbf{r}_{1}, \mathbf{r}_{2}\right)\left|\frac{e^{2}}{\varepsilon_{\infty} r_{12}}\right| \chi^{0}\left(\mathbf{r}_{1}, \mathbf{r}_{2}\right)>- \\
-\frac{i e \hbar H_{z}}{2 m^{*} c}<\chi^{0}\left(\mathbf{r}_{1}, \mathbf{r}_{2}\right)\left|\sum_{j=1,2}\left(x_{j} \frac{\partial}{\partial y_{j}}-y_{j} \frac{\partial}{\partial x_{j}}\right)\right| \chi^{0}\left(\mathbf{r}_{1}, \mathbf{r}_{2}\right)>
\end{gathered}
$$

We expand $\varepsilon[\chi]$ in power of $\mathbf{V}_{z}$ and keeping only the quadratic terms in the series, obtain

$$
\begin{gathered}
\varepsilon[\chi]=-\frac{\hbar^{2}}{2 m^{*}}<\chi^{0}\left(\mathbf{r}_{1}, \mathbf{r}_{2}\right)\left|\sum_{j=1,2} \nabla_{j}^{2}\right| \chi^{0}\left(\mathbf{r}_{1}, \mathbf{r}_{2}\right)>+ \\
+\frac{e^{2} \mathrm{H}_{z}^{2}}{8 m^{*} c^{2}}<\chi^{0}\left(\mathbf{r}_{1}, \mathbf{r}_{2}\right)\left|\sum_{j=1,2}\left(x_{j}^{2}+y_{j}^{2}\right)\right| \chi^{0}\left(\mathbf{r}_{1}, \mathbf{r}_{2}\right)>- \\
-\frac{i e \hbar \mathrm{H}_{z}}{2 m^{*} c}<\chi^{0}\left(\mathbf{r}_{1}, \mathbf{r}_{2}\right)\left|\sum_{j=1,2}\left(x_{j} \frac{\partial}{\partial y_{j}}-y_{j} \frac{\partial}{\partial x_{j}}\right)\right| \chi^{0}\left(\mathbf{r}_{1}, \mathbf{r}_{2}\right)>+ \\
+<\chi^{0}\left(\mathbf{r}_{1}, \mathbf{r}_{2}\right)\left|\frac{e^{2}}{\varepsilon_{\infty} r_{12}}\right| \chi^{0}\left(\mathbf{r}_{1}, \mathbf{r}_{2}\right)>- \\
-i \hbar \mathrm{v}_{z}<\chi^{0}\left(\mathbf{r}_{1}, \mathbf{r}_{2}\right)\left|\sum_{j=1,2} \frac{\partial}{\partial z_{j}}\right| \chi^{0}\left(\mathbf{r}_{1}, \mathbf{r}_{2}\right)>- \\
-\sum_{\mathbf{q}} \frac{\left|V_{\mathbf{q}} \rho_{\mathbf{q}}\right|^{2}}{\hbar \omega_{0}}+\mathrm{v}_{z}^{2} \sum_{\mathbf{q}}^{\left|q_{z} V_{\mathbf{q}} \rho_{\mathbf{q}}\right|^{2}} \frac{\hbar \omega_{0}^{3}}{2}+m^{*} \mathrm{v}_{z}^{2}= \\
=\varepsilon^{0}\left[\chi^{0}\right]+\frac{m^{* *} \mathrm{v}_{z}^{2}}{2}
\end{gathered}
$$
is

where the longitudinal trans lational mass of the bipolaron

$$
m^{* *}=2\left(m^{*}+\sum_{\mathbf{q}} \frac{\left|q_{z} V_{\mathbf{q}} \rho_{\mathbf{q}}\right|^{2}}{\hbar \omega_{0}^{3}}\right) \quad .
$$

Thus, for slow velocities, the energy of translational motion can be separated from the intrinsic energy of the polaron. This makes it possible to consider $\varepsilon^{0}\left[\chi^{0}\right]$ separately in Eq. (15). It is convenient henceforth to change from the $\mathbf{q}$ representation in Eq. (15) to a coordinate representation, and to introduce the one-electron spinless density function, which in the general case of an $N$-e lectron system may be written

$$
\rho_{1}\left(\mathbf{r}_{1}, \mathbf{r}_{1}^{\prime}\right)=N \int \chi^{0}\left(\mathbf{r}_{1}, \mathbf{r}_{2}, \ldots \mathbf{r}_{N}\right) \chi^{0}\left(\mathbf{r}_{1}^{\prime}, \mathbf{r}_{2}, \ldots \mathbf{r}_{N}\right) d \tau_{2} \ldots d \tau_{N},
$$

with $\rho_{1}\left(\mathbf{r}_{1}\right) \equiv \rho_{1}\left(\mathbf{r}_{1}, \mathbf{r}_{1}\right)$, and the two-electron spinless density function is

$$
\rho_{2}\left(\mathbf{r}_{1}, \mathbf{r}_{2}\right)=N(N-1) \int\left|\chi^{0}\left(\mathbf{r}_{1}, \mathbf{r}_{2}, \ldots \mathbf{r}_{N}\right)\right|^{2} d \tau_{3} \ldots d \tau_{N} .
$$

Then the self-consistent total energy of the stationary bipolaron formation may be written as follows:

$$
\begin{gathered}
\varepsilon^{0}\left[\chi^{0}\right]=-\frac{\hbar^{2}}{2 m^{*}} \int_{\left(\tau_{j}=\tau_{j}^{\prime}\right.} \sum_{j=1,2} \nabla_{j}^{2} \rho_{1}\left(\mathbf{r}_{j}, \mathbf{r}_{j}^{\prime}\right) d \tau_{j}+\frac{M_{L} \hbar \omega_{c}}{2}+ \\
+\frac{1}{4} \iint d \tau_{1} d \tau_{2} \rho_{2}\left(\mathbf{r}_{1}, \mathbf{r}_{2}\right) \times \\
\times\left\{\frac{2 g\left(\mathbf{r}_{1}, \mathbf{r}_{2}\right)}{\varepsilon_{\infty}}-\frac{1}{\varepsilon^{*}} \sum_{j=1,2} \int \rho_{1}\left(\mathbf{r}_{j}^{\prime}\right) g\left(\mathbf{r}_{j}, \mathbf{r}_{j}^{\prime}\right) d \tau_{j}^{\prime}\right\}+ \\
+\frac{m^{*} \omega_{0}^{2}}{16} \sum_{j=1,2} \int \rho_{1}\left(\mathbf{r}_{j}\right)\left(x_{j}^{2}+y_{j}^{2}\right) d \tau_{j},
\end{gathered}
$$

where $M_{L}$ is the projection of the orbital angular momentum $L$ on the selected axis $z$, the cyclotron frequency is $\omega_{c}=e \mathrm{H}_{z} / m^{*} c$, the electron-electron interaction operator is $g\left(\mathbf{r}_{1}, \mathbf{r}_{2}\right)=e^{2} /\left|\mathbf{r}_{1}-\mathbf{r}_{2}\right|$, and the energy is reckoned from the bottom of the conduction band at $\mathrm{H}_{z}=0$. In the derivation of Eq. (19), the identity

$$
\left|\mathbf{r}_{1}-\mathbf{r}_{2}\right|^{-1}=\left(2 \pi^{2}\right)^{-1} \int \exp \left[i \mathbf{q}\left(\mathbf{r}_{1}-\mathbf{r}_{2}\right)\right] q^{-2} d \mathbf{q}
$$

was considered, and the operator transformations of Ref. [12] were used.

We shall consider triplet singly excited electronic states (for distances $R \rightarrow \infty$ the energy of the two polaron system corresponds to the $1 s+2 p_{z, x \pm i y}$ configuration). $R=\left|\mathbf{R}_{1}-\mathbf{R}_{2}\right|$ is the distance between centers of polarons gravity [1,9], while the vector $\mathbf{r}_{i}$ is associated with oscillation motion of electron in polarization potential well near $\mathbf{R}_{i}$. The two-electron wave function of the electronic excited state, approximating the eigenfunction $\chi\left(r_{1}, r_{2}\right)$ should be antisymmetric with respect to the transposition of both electrons orthogonal to the ground state. In the approximation of quasi-independent electrons, the electronic part of the two-center wave function (the centers of the potential wells are found at points $a$ and $b$, separated by distance $R$ ) will be written in the Heitler-London form

$$
\chi^{0}\left(r_{1}, r_{2}\right)=N\left[\chi_{1 s, a}\left(r_{1}\right) \chi_{2 p_{m}, b}\left(r_{2}\right)-\chi_{1 s, b}\left(r_{1}\right) \chi_{2 p_{m}, a}\left(r_{2}\right)\right],
$$

where $N$ is the normalization constant. Subscripts $a$ and $b$ refer to polarons located at $R_{1}$ and $R_{2}$, respectively. $\chi_{1 s}$ and $\chi_{2 p_{m}}$ are one-particle wave functions of the $1 s$ and $2 p_{m}$ states, and $m=z, x \pm i y$. The coordinate one-particle wave functions are chosen in the form of orthonormalized two-parameter Gaussian-type functions 


$$
\begin{gathered}
\chi_{1 s}=\left(\pi^{3} \alpha^{2} \beta^{4}\right)^{-1 / 4} \exp \left[-z^{2} / 2 \alpha^{2}-\left(x^{2}+y^{2}\right) / 2 \beta^{2}\right], \\
\chi_{p_{z}}=\left(2 / \pi^{3 / 2} \mu^{2} \gamma^{3}\right)^{1 / 2} z \exp \left[-z^{2} / 2 \gamma^{2}-\left(x^{2}+y^{2}\right) / 2 \mu^{2}\right], \\
m_{l}=0, \\
\chi_{p_{x \pm i y}}=\left(\pi^{3} \eta^{8} \xi^{2}\right)^{-1 / 4}(x \pm i y) \exp \left[-z^{2} / 2 \xi^{2}-\right. \\
\left.-\left(x^{2}+y^{2}\right) / 2 \eta^{2}\right], m_{l}= \pm 1 .
\end{gathered}
$$

The minimization of Eq. (19) must be carried out with respect to the parameters $\alpha, \beta, \gamma, \mu, \xi$ and $\eta$ for fixed values of $R$ and $\varepsilon^{*} / \varepsilon_{\infty}$.

The magnetic field removes the degeneracy with respect to the magnetic quantum number $M_{L}$ of bipolaron, and the total energy Eq. (19) for the states $2^{3} P_{z}\left(M_{L}=0\right)$ and $2^{3} P_{x \pm i y}\left(M_{L}= \pm 1\right)$ may be written in the form

$$
\begin{gathered}
\varepsilon^{0}\left(2^{3} P_{z}\right)=K^{\prime}-U_{1}^{\prime}+U_{2}^{\prime}+U_{3}^{\prime}, \\
\varepsilon^{0}\left(2^{3} P_{x \pm i y}\right)=K^{\prime \prime}-U_{1}^{\prime \prime}+U_{2}^{\prime \prime}+U_{3}^{\prime \prime} \pm \hbar \omega_{c} / 2 .
\end{gathered}
$$

The variational parameters can be found only numerically, and in the selfconsistent state the virial theorem should be satis fied. We introduce the scale transformation $r \rightarrow t r, R \rightarrow$ $t R, \chi(r) \rightarrow t^{3 / 2} \chi(t r)$ and then obtain for the function of the total energy (without magnetic field)

$$
\varepsilon^{0}(w)=t^{2} K(w)-t U_{1}(w)+t U_{2}(w)+t U_{3}(w), w=t R,
$$

where $K$ is the mean kinetic energy of electrons, $U_{1}$ is the mean energy of interaction between electrons exchanging phonons, $U_{2}$ is diamagnetic part of the energy, and the mean Coulomb interaction energy of electrons is $U_{3}$. Minimizing Eq. (22) with respect to $t$ and assuming $t=1$, we obtain the virial relation

$$
2 K(R)-U_{1}(R)+U_{2}(R)+U_{3}(R)+R d \varepsilon^{0}(R) / d R=0 .
$$

The virial relation Eq.(25) should be satisfied for all interpolaron distances.

The trip let bipolaron in $2^{3} P_{z, x \pm i y}$ state will be stable to adiabatic dissociation by two polarons with conservation of the total spin and orbital angular mo mentu $m$ if the binding energy

$$
\Delta \varepsilon^{0}(R)=\varepsilon_{1 s}^{0}(R \rightarrow \infty)+\varepsilon_{2 p_{m}}^{0}(R \rightarrow \infty)-\varepsilon^{0}\left(2^{3} P_{z, x \pm i y} ; R\right)>0
$$

where $\varepsilon_{1 s}^{0}(R \rightarrow \infty)$ and $\varepsilon_{2 p_{m}}^{0}(R \rightarrow \infty)$ are the self-consistent total energy of the polarons in $1 s$ and $2 p_{m}$ states.

Figure 1 shows the total energy of the bipolaron on the distance $R$ for dielectric parameters $\varepsilon^{*} / \varepsilon_{\infty}=1.075$. Triplet bipolaron has minima when $R=0$. For $R=0$ in extreme case $\varepsilon_{s} \gg \varepsilon_{\infty}$ the ratio $Q=\varepsilon^{0}\left(2^{3} P_{z, x \pm i y}\right) /\left(\varepsilon_{1 s}^{0}+\varepsilon_{2 p_{m}}^{0}\right)=1.22$. With a decrease in the value of $Q$ ratio decreases and reaches unity at $\varepsilon^{*} / \varepsilon_{\infty}=1.15$ (or $\varepsilon_{s} / \varepsilon_{\infty}>7.67$ ).

Let us consider the change in the total energy of the triplet bipolaron in the limit of a weak magnetic field: $U_{3}<<U_{1}$. In this case, we assume that the bipolaron has central symmetry ( $\alpha=\beta$ and $\gamma=\mu=\xi=\eta$ ). Hence, to determine the energy change, use may be made of the methods of perturbation theory, which for the $2^{3} P_{z}$ state gives the energy correction

$$
\Delta\left(\Delta \varepsilon_{z}^{0}\right)=\Delta \varepsilon^{0}\left(2^{3} P_{z} ; H_{z} \neq 0\right)-\Delta \varepsilon^{0}\left(2^{3} P_{z} ; H_{z}=0\right)=
$$

$$
=\frac{m^{*} \omega_{c}^{2}}{8}\left(\beta_{0}+\beta\right)\left[\beta_{0}-\beta+\left(\mu_{0}-\mu\right)\left(\frac{\left.\mu_{0}+\mu\right)}{\beta_{0}+\beta}\right)\right]>0,
$$

since $\mu_{0}-\mu>\beta_{0}-\beta$ and $\left(\mu_{0}+\mu\right) /\left(\beta_{0}+\beta\right)>1[10]$ it is evident that in the presence of a magnetic field $\Delta \varepsilon^{0}\left(2^{3} P_{z} ; \mathrm{H}_{z} \neq 0\right)>\Delta \varepsilon^{0}\left(2^{3} P_{z} ; \mathrm{H}_{z}=0\right)$, i.e., the term of the total energy of the triplet bipolaron becomes lower, and is stabilization takes place. $\beta_{0}, \mu_{0}$ and $\eta_{0}$ are the parameters for $\mathrm{H}_{z}=0$. The corresponding inequality can also be written for $2^{3} P_{x \pm i y}$ term:

$$
\begin{gathered}
\Delta\left(\Delta \varepsilon_{x \pm i y}^{0}\right)=\Delta \varepsilon^{0}\left(2^{3} P_{x \pm i y} ; \mathrm{H}_{z} \neq 0\right)-\Delta \varepsilon^{0}\left(2^{3} P_{x \pm i y} ; \mathrm{H}_{z}=0\right)= \\
=\frac{m^{*} \omega_{c}^{2}}{8}\left(\beta_{0}+\beta\right)\left[\beta_{0}-\beta+2\left(\eta_{0}-\eta\right)\left(\frac{\left.\eta_{0}+\eta\right)}{\beta_{0}+\beta}\right)\right]>0,
\end{gathered}
$$

since $\eta_{0}-\eta>\beta_{0}-\beta$ and $\left(\eta_{0}+\eta\right) /\left(\beta_{0}+\beta\right)>1$.

In magnetic fields (dimensionless) $\delta \leq 0.1 \cdot \hbar \omega_{c} / R y^{*}$, where $R y^{*}=m^{*} e^{4} / 2 \varepsilon^{* 2} \hbar^{2}=\alpha_{c}^{2} \hbar \omega_{0}$, the change in coupling energy for states with $M_{L}=0$ and $M_{L}= \pm 1$ is different, and independently of the ratio of the permittivities of the medium, always $\Delta\left(\Delta \varepsilon_{x \pm i y}^{0}\right)>\Delta\left(\Delta \varepsilon_{z}^{0}\right)$. However, in magnetic fields: $\delta \geq 0.1$, the energy change of the $2^{3} P_{z}$ term becomes more significant than that of the $2^{3} P_{x \pm i y}$ term, and for polar media with a s maller ratio of $\varepsilon^{*} / \varepsilon_{\infty}$, this shift takes place in lower fields.

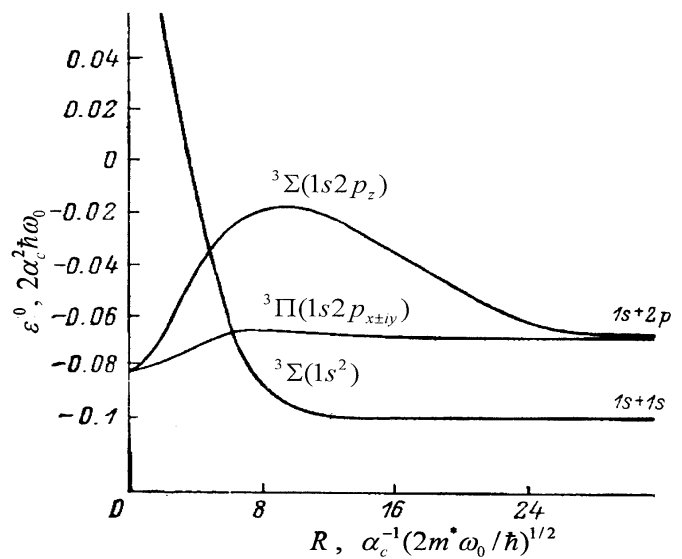

Figure 1. Triplet terms of the bipolaron as function of the polaron-polaron distance. $\varepsilon^{*} / \varepsilon_{\infty}=1.075$

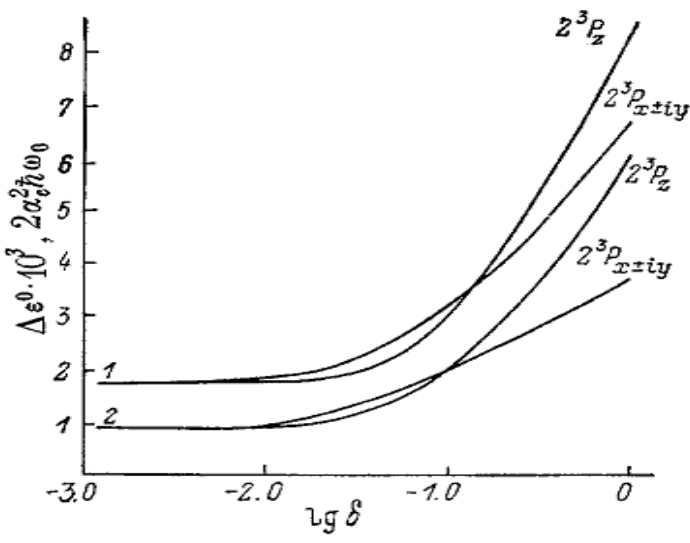

Figure 2. The binding energy of the triplet bipolaron as function of the magnetic field (dimensionless). $1-\varepsilon^{*} / \varepsilon_{\infty}=1.00,2-\varepsilon^{*} / \varepsilon_{\infty}=1.075$ 
In the range of magnetic field values for which $U_{1}>U_{3}$, i.e., $e \hbar \mathrm{H}_{z} / m^{*} c \leq 2 R y^{*}$, a variation calculation of the change in total energy terms is shown in Fig. 2 for two cases: $\varepsilon^{*} / \varepsilon_{\infty}=1.075$ and extreme ratio $\varepsilon^{*} / \varepsilon_{\infty}=1.00 \quad\left(\varepsilon_{s}>>\varepsilon_{\infty}\right)$. These results fully confirm the conclusions that follow from Eqs. (26) and (27). Irrespective of the permittivity ratio, a constant homogeneous magnetic field decreases the energy of the trip let bipolaron, and this in turn leads to stabilization of bipolarons with respect to dissociative decay. Moreover, the action of the magnetic field expands the range of dielectric media where bound two-polaron formation can exist.

Thus for the $2^{3} P_{z}$ state, even in a dimensionless field as low as $\delta=0.02$, the limitation $\left(\varepsilon_{s} / \varepsilon_{\infty} \geq 7.7\right)$ on the dielectric parameters of medium is reduced and will be $\varepsilon_{s} / \varepsilon_{\infty} \geq 7.2$, whereas for the $2^{3} P_{x \pm i y}$ state the effect of the magnetic field is still greater: $\varepsilon_{s} / \varepsilon_{\infty} \geq 6.2$.

Using the virial relation Eq. (25) is easy to show that the triplet bipolaron has spherical symmetry. Really, using the relation Eq. (25) and the condition $\Delta \varepsilon^{0}\left(R ; \mathrm{H}_{z}=0\right)>0$ we find

$$
V_{1}\left(1-\varepsilon^{*} / \varepsilon_{\infty}\right)+V_{2}>0 .
$$

Here we used the follow notation for two-center integrals:

$$
\begin{gathered}
V_{1}=<\chi_{2 p_{m}, b}^{2}\left(r_{1}\right)\left|g\left(\mathbf{r}_{1}, \mathbf{r}_{2}\right)\right| \chi_{1 s, a}^{2}\left(r_{2}\right)> \\
V_{2}=<\chi_{1 s, a}\left(r_{1}\right) \chi_{2 p_{m}, a}\left(r_{1}\right)\left|g\left(\mathbf{r}_{1}, \mathbf{r}_{2}\right)\right| \chi_{1 s, b}\left(r_{2}\right) \chi_{2 p_{m}, b}\left(r_{2}\right)>.
\end{gathered}
$$

In addition we assumed that for rough estimates of the one-electron wave functions of the polaron and bipolaron are the same (this gives the underestimation of the binding energy).

Hence the requirement of positive $\Delta \varepsilon^{0}(R)$ of Eq. (28) reduces to the inequality $\varepsilon^{*} / \varepsilon_{\infty}<1+V_{2} / V_{1}$. Using numerical values for $V_{1}$ and $V_{2}$ we obtain $\varepsilon^{*} / \varepsilon_{\infty}=1.17$ which is found in accordance with the result of the variational calculations of the triplet bipolaron. For the case $R \neq 0$ near zero, when $S=$ $<\chi_{1 s, a}(r) \mid \chi_{2 p_{m}, b}(r)><<1$, the inequality $\Delta \varepsilon^{0}\left(R>0 ; \mathrm{H}_{z}=0\right)<$ $\Delta \varepsilon^{0}\left(R=0 ; \mathrm{H}_{z}=0\right)$ is satisfied if

$$
V_{2} / V_{1}-2\left(V_{3}+V_{4}\right) / S V_{1}<\varepsilon^{*} / \varepsilon_{\infty}
$$

which is a lways valid, since $V_{1}>V_{2}>0, V_{3} \approx S V_{1}, V_{4} \approx S V_{2}$ and $S$ also are all positive. Here

$$
\begin{aligned}
& V_{3}=<\chi_{1 s, a}\left(r_{1}\right) \chi_{2 p_{m}, b}\left(r_{1}\right)\left|g\left(\mathbf{r}_{1}, \mathbf{r}_{2}\right)\right| \chi_{1 s, a}^{2}\left(r_{2}\right)>, \\
& V_{4}=<\chi_{2 p_{m}, b}^{2}\left(r_{1}\right)\left|g\left(\mathbf{r}_{1}, \mathbf{r}_{2}\right)\right| \chi_{1 s, a}\left(r_{2}\right) \chi_{2 p_{m}, b}\left(r_{1}\right)>.
\end{aligned}
$$

Thus, it has been shown that $R=0$ is really the total energy minimum. Consequently, in contrast to the singlet axialsymmetric bipolarons $[9,10]$ electron-excited trip let bipolarons are spherically symmetrical formations.

\section{Interelectron Correlations}

We now show that the inclusion of interelectron correlation leads to deeper potential well of the trip let bipolaron and extension of the dielectric media in which such formation may be exist. In the Hartree-Fock $(H F)$ approximation for electrons with parallel spin correlation is partially accounted. However, this approximation not taken into account intershell Coulomb correlation. To account for this correlation, we need to introduce explicitly the interelectronic distance $r_{12}$ in the symmetrized two-e lectron wave function

$$
\chi\left(r_{1}, r_{2}\right)=N\left(1+\gamma r_{12}^{2}\right) \sum_{M}(-1)^{p} M \chi_{1 s}\left(r_{1}\right) \chi_{2 p}\left(r_{2}\right) \chi^{T}\left(s_{1}, s_{2}\right)(30)
$$

here $\chi^{T}\left(s_{1}, s_{2}\right)$ is antisymmetric spin-function, $M$ is the operator of electron coordinates permutation, $p$ is number of individual pairs of transpositions in the permutation $M, \gamma$ is the additional variational parameter. As shown by calculations for extreme case $\varepsilon_{s}>>\varepsilon_{\infty}, \alpha_{c}>10$ parameter $\gamma=-0.994 \cdot 10^{-3} \alpha_{c}^{2}\left(\omega_{0} m^{*} / \hbar\right)$. We make up the ratio for the self-consistent total energies. $\varepsilon^{0}($ corr $) / \varepsilon^{0}(H F)=1.025$ (magnetic field $\mathrm{H}_{z}=0$ ). That is, the electron-electron correlations leads to a decrease in the total energy of the trip let bipolaron in comparis on with the Hartree-Fock approximation and the expansion of the existence of the triplet bipolaron. Macroscopic dielectric constants must now satisfy the weaker inequality $\varepsilon_{s} / \varepsilon_{\infty}>6.3$, than in the case of quasi-independent electrons $\varepsilon_{s} / \varepsilon_{\infty}>7.7$.

An alternative method of accounting electronic correlations is the series enhancement of electronic part of the wave function of the electronic configurations of the same symmetry (multiplicity). Assuming that the total spin $S$ is a constant of the motion, we expand the electronic wave function of the $2^{3} P_{z, x+y}$ term of the system of eigenfunctions of a centrally symmetric field

$$
\begin{aligned}
& \Psi\left(r_{1}, r_{2}\right)=\sum_{n_{1}^{l}, n_{2}^{l}>n_{1}^{l}} \sum_{l} A_{n_{1}^{l} n_{2}^{l}} P_{l}\left(\cos \vartheta_{12}\right) \times \\
& \times \sum_{M}(-1)^{p} M R_{n_{1}^{l} l}\left(r_{1}\right) R_{n_{2}^{l} l}\left(r_{2}\right) \chi^{T}\left(s_{1}, s_{2}\right),
\end{aligned}
$$

where $\vartheta_{12}$ is the angle between the radius vectors of the first and second electrons.

For examp le, take into account the superposition Eq. (31), two excited elecronic configurations $1 s 2 p$ and $2 s 2 p$. Moreover, the radial part of the single-partic le $2 s$ function chosen in the quasi-Coulo mb form $R_{20}(r) \sim r(1-\mu r)(1+\mu r) \exp (-\mu r)$.

Extremalization two-particle functional (2) under the additional condition of orthonormality of electronic configurations leads to the next value of self-consistent total energy (for the extreme case $\left.\varepsilon_{s}>\varepsilon_{\infty}, \quad \alpha_{c}>10\right): \varepsilon^{0}($ corr $)=-0.1812 \alpha_{c}^{2} \hbar \omega_{0}$ when the values of parameters are optimal $\alpha=0.615 \alpha_{c}\left(\omega_{0} m^{*} / \hbar\right)^{1 / 2}$, $\beta=0.615 \alpha_{c}\left(\omega_{0} m^{*} / \hbar\right)^{1 / 2}, \mu=0.194 \alpha_{c}\left(\omega_{0} m^{*} / \hbar\right)^{1 / 2}$, and mixing ratio $A_{1^{0} 2_{1}}=0.9940, A_{2^{0} 2_{1}^{1}}=-0.1093$. Dimensionless ratio $\varepsilon^{0}($ corr $) / \varepsilon^{0}(H F)=$ 1.02. That is already in the approximation of two configurations of most of the correlation energy can be accounted for. Lowering the total energy of the triplet bipolaron $2^{3} P_{z, x \pm i y}$ by electron-electron correlation has important consequences, which leads to enhancement of permissible limits of its existence. Macroscopic dielectric constants must now satisfy a somewhat weaker inequality $\varepsilon_{s} / \varepsilon_{\infty}>6.3$ than $\varepsilon_{s} / \varepsilon_{\infty}>7.5$ 
that was obtained without taking into account inter-shell electron correlation[1]. It should be noted that in the range $\varepsilon_{s}>>\varepsilon_{\infty}$ of the continual polaron theory methods are applicable, if the dimensionless ratio $m \varepsilon_{\infty} / m^{*}>0.5$ [1]. This inequality limits the range of existence of bipolaron formation, giving an upper bound for the dynamic polarizability. At the same time test the applicability of the adiabatic approximation $\left(m^{*} / m \varepsilon^{*}\right) \mathrm{eV}>>\hbar \omega_{0}$ holds the better, the higher the ratio $\varepsilon_{s} / \varepsilon_{\infty}$.

\section{Conclusions}

We establish conditions for the existence of electronically excited triplet bipolarons. It is shown that under certain conditions imposed on the macroscopic parameters of the dielectric medium, there may be quasi-stationary two- electron bipolaron formation in the triplet spin state. In contrast to the singlet bipolarons, triplet bipolarons are spherically symmetric. Similarly, the trip let bipolaron in $2^{3} \mathrm{P}$ state can exist triplet $2^{3} \mathrm{~S}$ bipolaron. In this case, one electron occupies the $1 \mathrm{~s}$ single-electron level and the other electron occupies the $2 \mathrm{~s}$ one-electron level[10]. However, the triplet term in the energy scale is lower than the initial single-particle states. The prohibition on the electron spin makes it impossible for the radiative deactivation of electron-excited triplet formation. Nonradiative deactivation is not effective also[10]. The action of a uniform magnetic field stabilizes the triplet bipolaron.

According to estimates [10], the nonradiative decay of the triplet bipolaron is of order $\left(\tau \approx 10^{6} \omega_{0}^{-1}\right)$, which is considerably longer than the characteristic phonon time $\left(\approx \omega_{0}^{-1}\right)$, as well as time $\left(\approx \alpha_{c}^{-2} \omega_{0}^{-1}\right)$ electron oscillations in the bipolaron potential well. The damping of the trip let bipolaron $\left(\gamma=\tau^{-1}\right)$ due to phonon emission decreases rapidly with increasing $\alpha_{c}$. Thus, the trip let bipolaron for times of order $\approx 10^{6} \omega_{0}^{-1}$ can be regarded as an equilibrium. In principle such triplet electron-excited bipolarons being the quasi-stationary formations, but with a suffic iently long lifetime, can transfer the trapped energy in a uniform electric field in the selected direction.

\section{REFERENCES}

[1] V. K. Mukhomorov, "Singlet and triplet states of a continual bipolaron for adiabatic and strong coupling," Sov. Phys. Optics and Spectroscopy, vol. 74, pp. 644-655, June 1993.

[2] S. I. Pekar. Research in Electron Theory of Crystals. US AEC Report AEC-tr-5575, Ed. USA, 1963.

[3] V. K. Mukhomorov, "Singlet and triplet states of quasimolecular dimer solvated in ammonia," Sov. Phys. - Optics and Spectroscopy., vol. 69, pp.43-46, July 1990.

[4] G. Iadonisi, M. Chiofolo, V. Cataudella, D. Ninno, "Phonon-plasmon cooperative effects in the dilute large-bipolaron gas: A possible mechanism for hogh- $\mathrm{T}_{\mathrm{c}}$ superconductivity," Phys. Rev. B, vol. 48, pp. 12966-12978, Nov. 1993-I.

[5] S. Sahoo, "A variational calculation of the stability of the two centre Fröhlich bipolaron," Phys. Lett. A, vol. 195, pp. 105-109, Sept. 1994.

[6] W. B. da Costa, P. M. Peeters, "Phase diagram for large two-dimensional bipolarons in a magnetic field," Phys. Rev. B, vol.57, pp.10569-10575, M ay 1998-I.

[7] R. T. Senger, A. Ercelebi, "On the stability of Fröhlich bipolarons in spherical quantum dots," Eur. Phys. J. B, vol.26, pp.5549-5560, May 2002.

[8] V. K. Mukhomorov, "Stability of bipolarons, electron-electron correlations, the variational principle, and the virial theorem," Physics of the Solid State, v.48, pp.864-870, May 2006.

[9] R. T. Senger, B. Kozal, A. Chatterjee, A. Ercelebi, "Hartree-Fock approximation of bipolaron state in quantum dotes and wires," Eur. Phys. J. B, vol.78, pp.525-529, Dec. 2010.

[10] V. K. Mukhomorov, Bipolarons. (Structure. Properties). Ed. Saarbrücken, Germany: Lambert Academic Publishing, 2011.

[11] E. Kartheuser, R. Evrard, J. Devreese, "Mechanism of absorption of light by free continuum polarons," Phys. Rev. Lett., vol.22, pp.94-97, Jan. 1969.

[12] R.McWeeny, B.T.Sutcliffe, Methods of Molecular Quantum Mechanics. Ed. New York, USA: Academic Press, 1969. 